\title{
Transcranial Motor Evoked Potentials of Lower Limbs Can Prognosticate Ambulation in Hemiplegic Stroke Patients
}

\author{
Pyoungsilk Hwang, MD, Min Kyun Sohn, MD, PhD, Sungju Jee, MD, Hyunkeun Lee, MD \\ Department of Rehabilitation Medicine, Chungnam National University School of Medicine, Daejeon, Korea
}

\begin{abstract}
Objective To examine the association between motor evoked potentials (MEPs) in lower limbs and ambulatory outcomes of hemiplegic stroke patients.

Methods Medical records of hemiplegic patients with the first ever stroke who received inpatient rehabilitation from January 2013 to May 2014 were reviewed. Patient who had diabetes, quadriplegia, bilateral lesion, brainstem lesion, severe musculoskeletal problem, and old age over 80 years were excluded. MEPs in lower limbs were measured when they were transferred to the Department of Rehabilitation Medicine. Subjects were categorized into three groups (normal, abnormal, and absent response) according to MEPs findings. Berg Balance Scale (BBS) and Functional Ambulation Category (FAC) at initial and discharge were compared among the three groups by one-way analysis of variance (ANOVA). Correlation was determined using a linear regression model.

Results Fifty-eight hemiplegic patients were included. BBS and FAC at discharge were significantly (ANOVA, $\mathrm{p}<0.001)$ different according to MEPs findings. In linear regression model of BBS and FAC using stepwise selection, patients' age ( $\mathrm{p}<0.01)$, BBS at admission $(\mathrm{p}<0.01)$, and MEPs $(\mathrm{p}<0.01)$ remained significant covariates. In regression assumption model of BBS and FAC at admission, MEPs and gender were significant covariates. Conclusion Initial MEPs of lower limbs can prognosticate the ambulatory outcomes of hemiplegic patients.
\end{abstract}

Keywords Stroke, Motor evoked potentials, Prognosis, Gait, Hemiplegia

\section{INTRODUCTION}

Gait disturbance is one crucial physical disability in stroke patients [1]. The loss of walking ability may hamper stroke patients' independent mobility and their social activities [2]. Since ambulatory dysfunctions after stroke can adversely affect the posture and movement patterns of stroke patients in their daily activities, recovering ambulatory function is one essential objective in stroke rehabilitation programs. In addition, walking ability repre-

Received June 11, 2015; Accepted September 24, 2015

Corresponding author: Min Kyun Sohn

Department of Rehabilitation Medicine, Daejeon Regional Rehabilitation Center, Chungnam National University Hospital, 282 Munhwa-ro, Jung-gu, Daejeon 35015, Korea. Tel: +82-42-338-2460, Fax: +82-42-338-2461, E-mail: mksohn@cnu.ac.kr

ORCID: Pyoungsik Hwang (http://orcid.org/0000-0001-6677-1789); Min Kyun Sohn (http://orcid.org/0000-0002-2548-545X); Sungju Jee (http://orcid. org/0000-0002-9400-9609); Hyunkeun Lee (http://orcid.org/0000-0002-9306-6011).

(c) This is an open-access article distributed under the terms of the Creative Commons Attribution Non-Commercial License (http://creativecommons.org/ licenses/by-nc/4.0) which permits unrestricted noncommercial use, distribution, and reproduction in any medium, provided the original work is properly cited. Copyright $\odot 2016$ by Korean Academy of Rehabilitation Medicine 
senting the recovery of motor function in stroke patients can be used as an indicator of their functional recovery. Early prediction for functional motor recovery in stroke patients can help the establishment of rehabilitation goals and treatment strategies.

In line with the use of motor evoked potentials (MEPs) in motor function-related neurophysiological testing, MEPs have also been used to predict motor function recovery in patients with cerebrovascular diseases [39]. MEPs are associated with the excitability of cerebral cortex. No response after proper magnetic stimulation indicates that neurons or neuronal stem cells are dead or higher than normal motor threshold $[10,11]$.

The recovery mechanism of muscular strength is through brain's response after injury. Increased synapses in intact areas can provide cortical reorganization or rewiring. Axonal sprouting and reorganization occur after denervation supersensitivity by brain plasticity [12] Clinical factors that may affect the recovery mechanism of muscular strength include age, gender, aphasia, comorbidity, visual disturbance, sensory disorder, mental and cognitive state, and motor functions [13,14]. Evoked potential testing has been implemented as one of objective techniques to obtain information regarding the injury and recovery of neurons. With the introduction of transcranial magnetic stimulation (TMS) by Barker [15] in 1985, a number of reports have indicated that noninvasive and painless MEPs obtained by TMS are useful early prognostic markers for motor function recovery by monitoring the response of limb muscles via directly stimulating the motor cortex $[3-5,16,17]$. However, few studies have analyzed motor recovery using a variety of clinical parameters such as age, gender, location and type of the lesion, gait ability at the time of admission, and MEPs testing. Therefore, the objective of this study was to determine the relationship between MEPs and the recovery of balance and ambulatory function in hemiplegic stroke patients. In addition, we analyzed the prognostic capacity of motor improvement based on the parameters such as age, gender, location and type of the lesion, and gait at the time of admission.

\section{MATERIALS AND METHODS}

\section{Subjects}

A total of 58 patients who were admitted to the Depart- ment of Rehabilitation Medicine in our hospital between January 2013 and May 2014 and diagnosed with the first onset of hemiplegic stroke were included in the study. Their medical records were retrospectively reviewed. The diagnosis of stroke based on medical history and physical exams was confirmed by brain computed tomography or magnetic resonance imaging. For inclusion, all patients had to meet the following criteria: initial onset of stroke, hemiplegia due to unilateral cerebral hemisphere lesion and supratentorial lesion. Exclusion criteria included known quadriplegia or lesion of bilateral cerebral hemisphere, legion of brain stem, and known history of stroke. Patients in coma, stupor, or acute confusion state were excluded from the study, including those who had cognitive impairment that disabled them from following test instructions given by medical staff. Other exclusion criteria included the presence of peripheral neuropathy that might affect MEPs responses and contraindications to TMS (seizure or coil embolization).

We reviewed the medical records of 215 stroke patients. After excluding recurrent stroke (48 patients), secondary upper motor neuron disease (21 patients), subcortical lesion (50 patients), lesion of bilateral cerebral hemisphere (36 patients), and secondary peripheral neuropathy (49 patients), a total of 58 patients were included in this study, including 32 males and 26 females with mean age of $61.0 \pm 14.5$ years. Of the 58 patients, 32 had cerebral infarction and 26 had hemorrhagic stroke. Twenty-one patients had lesion in cerebral cortex and 37 patients had subcortical lesions. Eighteen patients had stroke in the left hemisphere. The other 40 patients had stroke in the right hemisphere (Table 1).

\section{Electrophysiological evaluation}

Lower limb MEPs testing were performed for patients within a week after they were admitted or transferred to the Department of Rehabilitation Medicine in Daejeon Regional Rehabilitation Center, Chungnam National University Hospital. The mean duration between stroke onset and evaluation was $28.7 \pm 22.9$ days.

A MagPro X100 magnetic stimulator (MagVenture A/S, Farum, Denmark) was used for testing of MEPs. The motor of tibialis anterior muscle was triggered after stimulating motor cortex at the top of the head with a $126-\mathrm{mm}$ parabolic encircling coil (MMC-140-II). The intensity of magnetic stimulation was established at $80 \%$ of the maxi- 
Table 1. Demographic data of subjects $(n=58)$ used in this study

\begin{tabular}{lc}
\hline \multicolumn{1}{c}{ Variable } & Value \\
\hline Age (yr) & $61.0 \pm 14.5$ \\
Sex (male:female) & $32: 26$ \\
Lesion (cortex:subcortex) & $21: 37$ \\
Subtype of stroke (infarction:hemorrhage) & $32: 26$ \\
Hemiplegic side (left:right) & $18: 40$ \\
MEPs group (absent:abnormal:normal) & $17: 21: 20$ \\
Duration from onset to MEPs evaluation (day) & $28.7 \pm 22.9$ \\
Duration from onset to discharge (day) & $78.5 \pm 37.4$ \\
\hline
\end{tabular}

Values are presented as mean \pm standard deviation or number.

MEPs, motor evoked potentials.

mum intensity of the stimulator. MEPs responses were recorded on the convex site of the tibialis anterior muscle. Reference electrode was placed on the distant tendon of the tibialis anterior muscle. Magnetic stimulation was performed when the upper and lower extremities of a patient were relaxed. For MEPs testing, the applied band pass filter varied from $2 \mathrm{~Hz}$ to $10 \mathrm{kHz}$. Latency was measured from traces of stimulation to the first sound wave. Average latency was calculated from three consecutive tests. Normal latency was defined as less than $32.5 \mathrm{~ms}$. Delayed latency or abnormal latency was defined as equal to or more than $32.5 \mathrm{~ms}$. If the amplitude of recorded signals did not exceed $0.05 \mathrm{mV}$, MEPs were considered absent responses $[18,19]$. Based on MEPs outcomes, patients were divided into the following three groups: normal (17 patients), abnormal (21 patients), and absent response (20 patients).

\section{Clinical evaluation}

For the recovery of ambulatory function, scores of Berg Balance Scale (BBS) and Functional Ambulatory Category (FAC) were evaluated twice. One was at admission to the Department of Rehabilitation Medicine and the other was at the time of hospital discharge. Test results were assessed by a physical therapist of our hospital who was not informed about the MEPs outcomes.

\section{Berg balance scale}

BBS is a 14-item objective measure designed to assess static and dynamic balance. It has three categories: sitting, standing, and posture change. This tool has a
5 -point scale ranging from 0 to 4 . " 0 " indicates the lowest level of function while " 4 " indicates the highest level of function. The highest possible score was 56 points. The 14 items included sitting (sitting at correct posture unsupported), standing (standing unsupported, standing unsupported with eyes closed, turning to look behind over left and right shoulders while standing, pick up object from the floor from a standing position, standing unsupported with feet together, and reaching forward with outstretched arm while standing), and posture change (sitting to standing, standing to sitting, transfer from one chair to the other, turn $360^{\circ}$, and place alternative foot on step or stool while standing unsupported) $[20,21]$. The BBS not only has excellent inter-rater and intra-rater reliabilities in stroke patients $(\mathrm{r}=0.99$ and $\mathrm{r}=0.98$, respectively), but also has excellent internal consistency (Cronbach's $\alpha=0.97$ ) [21].

\section{Functional ambulatory category}

FAC was used to categorize the group of 58 patients according to basic motor skills necessary for functional ambulation. It consisted of the following 6 levels: 0 (patient ambulated only in parallel bars, or needed help from 2 or more persons), 1 (patient needed manual contact of one person during ambulation on level surfaces, and manual contact was continuous and necessary to support body weight and/or to prevent any fall), 2 (patient needed manual contact of one person during ambulation on level surfaces, and manual contact was continuous or intermittent light touch to prevent any fall), 3 (patient ambulated on level surfaces and required verbal supervision or stand-by help from one person because of poor judgment), 4 (patient could walk independently on level surfaces, but required help on slopes or stairs) and 5 (patient could walk independently on level surfaces, slopes, and stairs) [22,23].

\section{Statistical analysis}

All statistical analyses were performed using SPSS ver. 18.0 software (IBM Corp., Armonk, NY, USA). One-way analysis of variance (ANOVA) test was used to compare the differences in BBS and FAC values among the three groups divided by age and MEPs outcomes followed by Tukey's HSD (honestly significant difference) test. Independent sample t-test was used to compare differences in BBS and FAC values between sex, subtype of stroke, le- 
sion, and hemiplegic side.

Clinical parameters such as age, gender, location and type of the lesion, gait ability at the time of admission, and MEPs outcomes were studied by linear stepwise regression analysis. Statistical significance was considered when $\mathrm{p}$-value was less than 0.05 .

\section{RESULTS}

\section{Independent sample t-test and one-way ANOVA test}

Older group showed significantly poorer balance function at discharge than younger group. The female group showed significantly poorer balance function both at initial admission and at discharge with poorer ambulatory function at initial admission than the male group. However, BBS and FAC had no significant $(p>0.05)$ difference according to subtype of stroke or lesion side. Left hemiplegic patients presented poorer balance and am- bulatory function than right hemiplegic patients both at initial admission and at discharge (Table 2).

Significant differences in both BBS and FAC values both at admission and at discharge were observed among the three MEPs groups: BBS $(\mathrm{F}=17.7, \mathrm{p}<0.01)$ and FAC $(\mathrm{F}=16.0, \mathrm{p}<0.01)$ at admission, $\mathrm{BBS}(\mathrm{F}=23.8, \mathrm{p}<0.01)$ and FAC $(\mathrm{F}=33.4, \mathrm{p}<0.01)$ at discharge. However, one-way ANOVA test did not yield significant difference in delta BBS or delta FAS among the three groups (delta BBS or delta FAS were differences between the scores measured at admission and at discharge), suggesting insignificant differences in clinical improvement of balance and gait ability among the three groups (Table 2).

For patients who could walk independently and were scored 56 points in BBS score and 5 points in FAC score at admission, the two scores at discharge did not show significant changes when compared to those at admission. For this reason, 50 patients were again studied by

Table 2. BBS and FAC values at initial admission and at discharge, delta BBS, and delta FAC values of subjects ( $\mathrm{n}=58$ )

\begin{tabular}{|c|c|c|c|c|c|c|c|}
\hline \multirow{2}{*}{ Variable } & \multirow{2}{*}{ No. } & \multicolumn{2}{|c|}{ Initial } & \multicolumn{2}{|c|}{ Discharge } & \multicolumn{2}{|c|}{ Delta } \\
\hline & & BBS & FAC & BBS & FAC & BBS & FAC \\
\hline \multicolumn{8}{|l|}{ Age (yr) } \\
\hline $18-39$ & 4 & $27.5 \pm 23.9$ & $1.5 \pm 1.9$ & $48.5 \pm 6.0^{\mathrm{b})}$ & $3.5 \pm 1.0$ & $21.0 \pm 20.9$ & $2.0 \pm 1.2$ \\
\hline $40-59$ & 23 & $26.6 \pm 22.0$ & $1.9 \pm 1.5$ & $44.1 \pm 15.7^{\mathrm{b})}$ & $3.6 \pm 1.2$ & $17.5 \pm 18.5$ & $1.7 \pm 0.9$ \\
\hline $60-80$ & 31 & $15.0 \pm 17.9$ & $1.1 \pm 1.3$ & $30.2 \pm 21.2^{\mathrm{b})}$ & $2.7 \pm 1.7$ & $15.2 \pm 13.3$ & $1.6 \pm 1.2$ \\
\hline \multicolumn{8}{|l|}{ Sex } \\
\hline Male & 32 & $26.0 \pm 22.3^{\mathrm{a})}$ & $1.8 \pm 1.5^{\mathrm{a})}$ & $41.5 \pm 17.8^{\mathrm{a})}$ & $3.5 \pm 1.4$ & $15.5 \pm 15.9$ & $1.7 \pm 0.9$ \\
\hline Female & 26 & $13.7 \pm 15.9^{\text {a) }}$ & $1.0 \pm 1.2^{\mathrm{a})}$ & $31.4 \pm 20.8^{\mathrm{a})}$ & $2.7 \pm 1.5$ & $17.7 \pm 16.1$ & $1.7 \pm 1.3$ \\
\hline \multicolumn{8}{|c|}{ Subtype of stroke } \\
\hline Infarction & 32 & $20.0 \pm 20.1$ & $1.3 \pm 1.4$ & $38.2 \pm 19.3$ & $3.2 \pm 1.5$ & $18.1 \pm 16.3$ & $1.9 \pm 1.1$ \\
\hline Hemorrhage & 26 & $20.9 \pm 21.4$ & $1.6 \pm 1.5$ & $35.5 \pm 20.4$ & $3.0 \pm 1.6$ & $14.6 \pm 15.5$ & $1.4 \pm 0.9$ \\
\hline \multicolumn{8}{|l|}{ Lesion } \\
\hline Cortex & 21 & $19.0 \pm 21.9$ & $1.5 \pm 1.6$ & $37.0 \pm 20.0$ & $3.3 \pm 1.5$ & $18.0 \pm 17.5$ & $1.8 \pm 1.3$ \\
\hline Subcortex & 37 & $21.3 \pm 20.0$ & $1.4 \pm 1.4$ & $37.0 \pm 19.8$ & $3.0 \pm 1.5$ & $15.6 \pm 15.0$ & $1.6 \pm 0.9$ \\
\hline \multicolumn{8}{|l|}{ Hemiplegic side } \\
\hline Left & 18 & $14.1 \pm 16.4^{\mathrm{a})}$ & $0.9 \pm 1.0^{\mathrm{a})}$ & $31.8 \pm 22.7^{\mathrm{a})}$ & $2.7 \pm 1.8^{\mathrm{a})}$ & $17.7 \pm 15.8$ & $1.8 \pm 1.3$ \\
\hline Right & 40 & $23.4 \pm 21.7^{\mathrm{a})}$ & $1.7 \pm 1.5^{\mathrm{a})}$ & $39.3 \pm 18.0^{\mathrm{a})}$ & $3.3 \pm 1.3^{\mathrm{a})}$ & $16.0 \pm 16.1$ & $1.6 \pm 1.0$ \\
\hline \multicolumn{8}{|l|}{ MEPs group } \\
\hline Absent & 7 & $7.0 \pm 10.0^{\mathrm{b})}$ & $0.4 \pm 0.6^{b)}$ & $19.4 \pm 17.8^{b)}$ & $1.6 \pm 1.3^{\mathrm{b})}$ & $12.4 \pm 15.4$ & $1.2 \pm 1.1$ \\
\hline Abnormal & 21 & $15.2 \pm 16.9^{b)}$ & $1.2 \pm 1.3^{\mathrm{b})}$ & $36.1 \pm 16.5^{b)}$ & $3.2 \pm 1.0^{\mathrm{b})}$ & $20.9 \pm 15.1$ & $2.0 \pm 1.0$ \\
\hline Normal & 20 & $37.4 \pm 19.6^{\mathrm{b})}$ & $2.5 \pm 1.4^{\mathrm{b})}$ & $52.8 \pm 8.5^{\mathrm{b})}$ & $4.4 \pm 0.8^{\mathrm{b})}$ & $15.4 \pm 15.9$ & $1.8 \pm 1.1$ \\
\hline
\end{tabular}

Values are presented as mean \pm standard deviation.

MEPs, motor evoked potentials; BBS, Berg Balance Scale; FAC, Functional Ambulatory Category.

${ }^{a)} \mathrm{p}<0.05$ by independent $\mathrm{t}$-test.

${ }^{\text {b) }} \mathrm{p}<0.05$ by one-way ANOVA test. 
one-way ANOVA test after excluding 8 patients who were independently ambulant and rated at $4-5$ points in FAC score at admission. As a result, there were clinical improvements in balance and gait ability in terms of BBS value $(\mathrm{F}=9.9, \mathrm{p}<0.01)$ and $\mathrm{FAC}$ value $(\mathrm{F}=10.9, \mathrm{p}<0.01)$ at admission as well as $\mathrm{BBS}$ value $(\mathrm{F}=16.6, \mathrm{p}<0.01)$ and $\mathrm{FAC}$ value $(\mathrm{F}=22.9, \mathrm{p}<0.01)$ at discharge. In addition, there were significant differences in delta FAC $(\mathrm{F}=5.7, \mathrm{p}<0.01)$ that was obtained by subtracting the values measured at admission from those measured at discharge (Table 3).

\section{Linear regression analysis}

In an attempt to assess clinical parameters that might affect balance and gait ability of patients at discharge, linear regression analysis was conducted using parameters such as age, gender, location and type of the lesion, loca-

Table 3. Descriptive statistics and analysis of variance in subjects with initial FAC $<4(n=50)$

\begin{tabular}{lccl}
\hline & \multicolumn{3}{c}{ MEPs group } \\
\cline { 2 - 4 } & Absent & Abnormal & Normal \\
\hline BBS & & & \\
Initial $^{\text {a) }}$ & $7.0 \pm 10.0$ & $12.7 \pm 14.8$ & $29.9 \pm 18.9$ \\
Discharge $^{\text {a) }}$ & $19.4 \pm 17.8$ & $35.7 \pm 16.4$ & $51.4 \pm 9.9$ \\
Delta & $12.4 \pm 15.4$ & $23.1 \pm 14.2$ & $21.5 \pm 16.5$ \\
FAC $_{\text {Initial }}{ }^{\text {a) }}$ & $0.4 \pm 0.6$ & $1.0 \pm 0.9$ & $1.9 \pm 1.0$ \\
Discharge $^{\text {a) }}$ & $1.6 \pm 1.3$ & $3.1 \pm 0.9$ & $4.1 \pm 0.8$ \\
Delta $^{\text {a) }}$ & $1.2 \pm 1.1$ & $2.1 \pm 0.9$ & $2.2 \pm 1.0$ \\
\hline
\end{tabular}

Values are presented as mean \pm standard deviation. MEPs, motor evoked potentials; BBS, Berg Balance Scale; FAC, Functional Ambulation Category.

${ }^{a)} \mathrm{p}<0.05$ by one-way ANOVA. tion of hemiplegia, balance and gait ability at admission, and MEPs outcomes as independent variables. Influential factors on both BBS and FAC values at the time of discharge included age $(\mathrm{p}<0.01)$, BBS or FAC values $(\mathrm{p}<0.01)$ at admission, and MEPs outcomes $(p<0.01)$. There was negative correlation between age and BBS or FAC with older group showing poorer balance and ambulatory function at discharge. Categorized MEPs showed positive correlation with BBS or FAC with the balance and ambulatory function being the poorest in the absent response MEPs group at discharge (Table 4).

Linear stepwise regression analysis was performed to estimate the regression model on BBS and FAC values at discharge and changes in BBS and FAC values between admission and discharge by using clinical parameters such as age, gender, location and type of the lesion, BBS and FAC values at admission, and classified MEPs outcomes as independent variables. In model I, initial BBS and FAC was used as the significant variable, followed by addition of MEPs outcome in model II and age in model III. Changes in BBS and FAC values between admission and discharge showed negative correlations with initial BBS and FAC values (Table 5).

In our last analysis of regression equation estimation model on BBS and FAC values at admission, MEPs outcomes in model I were closely associated with better balance and gait ability when gender was included for analysis in models II and III. The balance and gait ability in female patients were worse than those in male patients at admission (Table 6).

Since the duration from stroke onset to MEPs evaluation was heterogeneous among the study subjects, we analyzed a subgroup (subacute) of patients who had

Table 4. Variables affecting BBS and FAC at discharge

\begin{tabular}{lcccc}
\hline \multirow{2}{*}{ Variable } & \multicolumn{2}{c}{ Discharge BBS } & \multicolumn{2}{c}{ Discharge FAC } \\
\cline { 2 - 5 } & $\boldsymbol{\beta}$ & $\mathbf{p}$-value & $\boldsymbol{\beta}$ & p-value \\
\hline Age & -0.39 & $0.00^{\mathrm{a}}$ & -0.28 & $0.00^{\mathrm{a})}$ \\
Sex & -0.07 & 0.41 & -0.10 & 0.19 \\
Lesion & 0.09 & 0.31 & 0.01 & 0.93 \\
Kind of stroke & -0.10 & 0.28 & -0.06 & 0.46 \\
Hemiplegic side & 0.02 & 0.83 & 0.05 & 0.52 \\
Admission BBS & 0.20 & $0.04^{\mathrm{a})}$ & 0.34 & $0.00^{\mathrm{a})}$ \\
\hline Categorized MEPs & 0.54 & $0.00^{\mathrm{a})}$ & 0.51 & $0.00^{\mathrm{a})}$ \\
\hline
\end{tabular}

MEPs, motor evoked potentials; BBS, Berg Balance Scale; FAC, Functional Ambulation Category.

${ }^{a)} \mathrm{p}<0.05$ by linear regression model. 
Table 5. Estimated regression model analysis of the BBS and FAC at discharge

\begin{tabular}{|c|c|c|c|c|c|c|c|}
\hline \multirow{2}{*}{ Model } & \multirow{2}{*}{ Variable } & \multicolumn{3}{|c|}{ Discharge BBS } & \multicolumn{3}{|c|}{ Delta BBS } \\
\hline & & $\beta$ & p-value & ${\text { Adjusted } \mathbf{R}^{2}}$ & $\beta$ & p-value & ${\text { Adjusted } \mathbf{R}^{2}}^{2}$ \\
\hline I & Initial BBS & 0.69 & 0.000 & 0.47 & 0.69 & 0.000 & 0.47 \\
\hline \multirow[t]{2}{*}{ II } & Initial BBS & 0.44 & 0.000 & 0.59 & 0.44 & 0.000 & 0.59 \\
\hline & Categorized MEPs & 0.42 & 0.000 & & 0.42 & 0.000 & \\
\hline \multirow[t]{4}{*}{ III } & Initial BBS & 0.23 & 0.026 & 0.71 & 0.23 & 0.026 & 0.71 \\
\hline & Categorized MEPs & 0.51 & 0.000 & & 0.51 & 0.000 & \\
\hline & Age & -0.39 & 0.000 & & -0.39 & 0.000 & \\
\hline & & \multicolumn{3}{|c|}{ Discharge FAC } & \multicolumn{3}{|c|}{ Delta FAC } \\
\hline I & Initial FAC & 0.74 & 0.000 & 0.54 & -0.31 & 0.016 & 0.08 \\
\hline \multirow[t]{2}{*}{ II } & Initial FAC & 0.46 & 0.000 & 0.67 & -0.70 & 0.000 & 0.34 \\
\hline & Categorized MEPs & 0.46 & 0.000 & & 0.64 & 0.000 & \\
\hline \multirow[t]{3}{*}{ III } & Initial FAC & 0.35 & 0.000 & 0.74 & -0.86 & 0.000 & 0.48 \\
\hline & Categorized MEPs & 0.51 & 0.000 & & 0.71 & 0.000 & \\
\hline & Age & -0.29 & 0.000 & & -0.40 & 0.000 & \\
\hline
\end{tabular}

Independent variables for estimated regression model: sex, age, lesion, kind of stroke, initial BBS or FAC, categorized MEPs.

By stepwise selection method.

BBS, Berg Balance Scale; FAC, Functional Ambulation Category; MEPs, motor evoked potentials; $\beta$, standardized coefficients.

Table 6. Estimated regression model analysis of the BBS and FAC at admission

\begin{tabular}{clccc}
\hline \multirow{2}{*}{ Model } & Variable & \multicolumn{3}{c}{ Initial BBS } \\
\cline { 3 - 5 } & Categorized MEPs & $\boldsymbol{\beta}$ & $\mathbf{p}$-value & Adjusted R $^{\mathbf{2}}$ \\
\hline I & Categorized MEPs & 0.60 & 0.000 & 0.35 \\
II & Age & 0.58 & 0.000 & 0.46 \\
& Categorized MEPs & -0.34 & 0.001 & 0.49 \\
\hline III & Age & 0.58 & 0.000 & \\
& Sex & -0.28 & 0.007 & 0.35 \\
& & -0.21 & 0.036 & 0.42 \\
\hline & Categorized MEPs & 0.60 & 0.000 & \\
\hline II & Categorized MEPs & 0.60 & 0.000 & \\
& Sex & -0.27 & 0.009 & \\
\hline
\end{tabular}

Independent variables for estimated regression model: sex, age, lesion, kind of stroke, categorized MEPs.

By stepwise selection method.

BBS, Berg Balance Scale; FAC, Functional Ambulation Category; MEPs, motor evoked potentials; $\beta$, standardized coefficients.

MEPs evaluation between 2 weeks and 3 months after the stroke onset. Using the same statistical analysis, the subacute group of patients showed the same results as the whole study subjects.

\section{DISCUSSION}

In the present study, we identified the relationship between TMS-based MEPs outcomes and the recovery of balance and gait ability in patients experiencing the 
first onset of hemiplegic stroke. We also analyzed the prognostic capacity of motor improvement using clinical parameters such as age, gender, location and type of the lesion, and gait parameters at the time of hospital admission.

On analysis of balance and gait ability at admission and discharge, significant differences were observed in balance and gait ability among the three MEPs groups. However, improved balance and gait ability during hospital admission had no significant change in either BBS or FAC values among the three groups. Under the notion that the above results might be due to a ceiling effect, we re-performed one-way ANOVA for 50 patients who were rated at lower than 4 points in FAC score at admission. The results revealed that there were significant differences in the improvement of FAC scores associated with MEPs outcomes among groups.

In our study, patients were subdivided into three groups (normal, abnormal, and absent response groups) by the difference in latency of MEPs. This enabled us to analyze the prognosis of balance and gait ability in stroke patients in more details. We also performed additional statistical analysis to clarify the ceiling effect, which has not been presented in previous studies.

Using linear regression analysis and estimated regression model, our results showed that the influential factors on both BBS and FAC values at discharge and their changes from admission to discharge included balance and gait ability, classified MEPs outcomes, and age at admission. Balance and gait ability at admission was the most influential single factor. When MEPs outcomes and age were incorporated into the model, a larger explanatory power was found. Van der Cruyssen et al. [24] has reported that age and physical disability following the onset of stroke is one of the most important prognostic factors in stroke patients. Nevertheless, the results of our study suggested that MEPs outcomes were the most influential factor, along with clinical parameters such as age and physical disability at the time of initial admission.

Interestingly, gender was found to be another influencing factor in estimated regression model on BBS and FAC values at admission. Our findings were consistent with the reports by Santalucia et al. [25] and Paolucci et al. [26] that the severity and function disorder of initial stroke in female patients was greater than those in male patients.

Our study has several limitations. First, this study had a small sample size (58 patients). When these patients were divided into three groups based on MEPs outcomes, there was a lack of statistical power. Second, irregular MEPs testing period of $28.7 \pm 22.9$ days (min, 5 days; max, 92 days) after stroke onset might affect the MEPs outcomes. Lastly, this was a retrospective study that we could not homogenously adjust types, time of rehabilitation treatment, or medications in stroke patients.

Despite the limitations detailed above, this study was meaningful as TMS-based MEPs testing allowed us to identify that the technique was useful for predicting the recovery of balance and gait ability in stroke patients. Based on the results of this study, clinicians could predict the functional prognosis in early stage after stroke and establish effective medical rehabilitation objectives based on predicted prognosis prior to treatment. However, additional prospective studies with larger patient populations with homogeneous nature must be conducted to ensure better reliability of our study findings.

After reviewing both BBS and FAC values at the time of admission and discharge for 58 new hemiplegic stroke patients who are divided into normal, abnormal, and absent response groups based on TMS-based MEPs outcomes, we have the following findings:

MEPs obtained by TMS are a useful prognostic markers for balance and gait ability in stroke patients depending on their age and physical disability at the time of admission. Patients with normal MEPs response findings at baseline showed the best improvement in balance and gait ability at discharge, followed by patients with abnormal and absent MEP response. Prognostic outcomes of younger patients following stroke onset were also better than those of older patients. Balance and gait ability in female patients were worse than those in male patients at the time of admission. However, factors such as cortical and/or subcortical lesions, types of cerebral hemorrhage and infarction, and left or right hemisphere of lesion did not influence the prognosis of patients.

Based on the results of this study, clinicians should predict the functional prognosis at an early stage after stroke and establish effective medical rehabilitation objectives based on predicted prognosis prior to treatment.

\section{CONFLICT OF INTEREST}

No potential conflict of interest relevant to this article was reported. 


\section{ACKNOWLEDGMENTS}

This study was supported by grants (HI13C1990, HI10C2020) of the Korean Health Technology R\&D Project funded by the Ministry of Health \& Welfare, Republic of Korea.

\section{REFERENCES}

1. Baer HR, Wolf SL. Modified emory functional ambulation profile: an outcome measure for the rehabilitation of poststroke gait dysfunction. Stroke 2001;32: 973-9.

2. Perry J, Garrett M, Gronley JK, Mulroy SJ. Classification of walking handicap in the stroke population. Stroke 1995;26:982-9.

3. Escudero JV, Sancho J, Bautista D, Escudero M, LopezTrigo J. Prognostic value of motor evoked potential obtained by transcranial magnetic brain stimulation in motor function recovery in patients with acute ischemic stroke. Stroke 1998;29:1854-9.

4. Rapisarda G, Bastings E, de Noordhout AM, Pennisi G, Delwaide PJ. Can motor recovery in stroke patients be predicted by early transcranial magnetic stimulation? Stroke 1996;27:2191-6.

5. Heald A, Bates D, Cartlidge NE, French JM, Miller S. Longitudinal study of central motor conduction time following stroke. 2. Central motor conduction measured within $72 \mathrm{~h}$ after stroke as a predictor of functional outcome at 12 months. Brain 1993;116(Pt 6): 1371-85.

6. Kang MJ, Yoon TS, Park CI, Chun SI. Motor evoked potential in stroke. J Korean Acad Rehabil Med 1993; 17:26-35.

7. Kang DW, Park SH, Lee YJ, Chun JU, Ko SB, Yoon BW, et al. Early transcranial magnetic stimulation can predict motor recovery of subacute stage in acute ischemic stroke patients. Korean J Stroke 2000;2:57-61.

8. Kim C, Jeong J. The significance of motor evoked potentials as a prognostic factor in the early stage of stroke patients. J Korean Acad Rehabil Med 1999;23: 1213-20.

9. Han TR, Bang MS, Lee KW. Motor evoked potentials of upper and lower extremities by magnetic stimulation in hemiparesis. J Korean Acad Rehabil Med 1998;22:386-91.
10. Jung HY, Kim TH, Park JH. Relationship of national institute of health stroke scale and motor evoked potentials in subjects with stroke. J Korean Acad Rehabil Med 2005;29:563-7.

11. Rossini PM, Barker AT, Berardelli A, Caramia MD, Caruso G, Cracco RQ, et al. Non-invasive electrical and magnetic stimulation of the brain, spinal cord and roots: basic principles and procedures for routine clinical application. Report of an IFCN committee. Electroencephalogr Clin Neurophysiol 1994;91:79-92.

12. Lee RG, van Donkelaar P. Mechanisms underlying functional recovery following stroke. Can J Neurol Sci 1995;22:257-63.

13. Allen CM. Predicting the outcome of acute stroke: a prognostic score. J Neurol Neurosurg Psychiatry 1984; 47:475-80.

14. Anderson TP. Studies up to 1980 on stroke rehabilitation outcomes. Stroke 1990;21(9 Suppl):II43-5.

15. Barker AT. An introduction to the basic principles of magnetic nerve stimulation. J Clin Neurophysiol 1991;8:26-37.

16. D'Olhaberriague L, Espadaler Gamissans JM, Marrugat J, Valls A, Oliveras Ley C, Seoane JL. Transcranial magnetic stimulation as a prognostic tool in stroke. J Neurol Sci 1997;147:73-80.

17. Macdonell RA, Donnan GA, Bladin PF. A comparison of somatosensory evoked and motor evoked potentials in stroke. Ann Neurol 1989;25:68-73.

18. Eisen AA, Shtybel W. AAEM minimonograph \#35: Clinical experience with transcranial magnetic stimulation. Muscle Nerve 1990;13:995-1011.

19. Lawrence RR. Magnetic stimulation of the central and peripheral nervous system. In: Dumitru D, Amato AA, Zwarts MJ, editors. Electrodiagnostic medicine. 2nd ed. Philadelphia: Hanley \& Belfus; 2002. p. 415-27.

20. Smith PS, Hembree JA, Thompson ME. Berg balance scale and functional reach: determining the best clinical tool for individuals post acute stroke. Clin Rehabil 2004;18:811-8.

21. Blum L, Korner-Bitensky N. Usefulness of the Berg balance scale in stroke rehabilitation: a systematic review. Phys Ther 2008;88:559-66.

22. Viosca E, Martinez JL, Almagro PL, Gracia A, Gonzalez C. Proposal and validation of a new functional ambulation classification scale for clinical use. Arch Phys Med Rehabil 2005;86:1234-8. 
23. Viosca E, Lafuente R, Martinez JL, Almagro PL, Gracia A, Gonzalez C. Walking recovery after an acute stroke: assessment with a new functional classification and the Barthel Index. Arch Phys Med Rehabil 2005; 86:1239-44.

24. Van der Cruyssen K, Vereeck L, Saeys W, Remmen R. Prognostic factors for discharge destination after acute stroke: a comprehensive literature review. Disabil Rehabil 2015;37:1214-27.
25. Santalucia P, Pezzella FR, Sessa M, Monaco S, Torgano G, Anticoli S, et al. Sex differences in clinical presentation, severity and outcome of stroke: results from a hospital-based registry. Eur J Intern Med 2013;24:16771.

26. Paolucci S, Bragoni M, Coiro P, De Angelis D, Fusco FR, Morelli $\mathrm{D}$, et al. Is sex a prognostic factor in stroke rehabilitation? A matched comparison. Stroke 2006; 37:2989-94. 